06

\title{
Измерение бесконтактным методом удельного электрического сопротивления листового металла в области высоких температур
}

\author{
(C) А.В. Румянцев, М.А. Никишин, А.П. Синицин
}

Балтийский фредеральный университет им. Иммануила Канта, 236041 Калининград, Россия

e-mail: albert37@list.ru

Поступило в Редакцию 23 октября 2019 г.

В окончательной редакции 25 марта 2020 г.

Принято к публикации 26 марта 2020 г.

Разработанный бесконтактный метод измерения удельного электрического сопротивления металлов в области высоких температур распространен на листовой металл. Теоретическая часть метода дополнена решением задачи о введенной в образец в виде тонкостенного цилиндра мощности при его индукционном нагреве. Суть метода - измерение электродвижущих сил (ЭДС), наведенных высокочастотным электромагнитным полем в двух круговых контурах разного диаметра, коаксиально расположенных с образцом цилиндрической формы. Полученная формула для мощности, введенной в тонкостенный цилиндр, позволила найти функцию, связывающую удельное электрическое сопротивление материала образца и ЭДС. По измеренным в эксперименте ЭДС определена величина удельного электрического сопротивления исследуемого образца и, как следствие, величина введенной в образец мощности. Последнее позволяет определить полусферическую интегральную степень черноты материала образца. Приведены результаты экспериментальной проверки метода на образце в виде тонкостенного цилиндра из ниобия. Полученные данные в пределах $3 \%$ согласуются с данными других авторов, что позволяет рекомендовать техническое использование метода. Приведены методики проведения эксперимента для реализации метода и обработки его результатов.

Ключевые слова: удельное электрическое сопротивление, электромагнитное поле, электродвижущая сила, скин-слой, листовой металл, высокая температура, тонкостенный цилиндр, мощность.

DOI: 10.21883/JTF.2020.10.49802.348-19

\section{Введение}

Основной недостаток контактного метода измерения удельного электрического сопротивления металлов, основанного на законе Ома, следует из его названия контактный. Если измерениям подлежит листовой металл толщиной $0.1-0.5 \mathrm{~mm}$, то осуществление надежного контакта образца в виде тонкостенного цилиндра с токоподводящими и потенциальными выводами представляет собой довольно сложную задачу, поскольку осуществить это можно только с помощью электроннолучевой сварки, отсутствующей, как правило, не в специализированных лабораториях. Вторая причина материал образца и проводов как токоподводящих, так и потенциальных (диаметрами $\leq 1.0 \mathrm{~mm}$ и $\leq 0.1 \mathrm{~mm}$ соответственно) должен быть одним и тем же, что далеко не всегда осуществимо. Существенным становится вопрос учета влияния теплоотвода в месте контакта потенциальных выводов с тонкостенным образцом, особенно когда диаметр проводов сопоставим с толщиной стенки исследуемого образца. В ряде случаев невозможно изготовить хорошие контакты, так как в процессе их изготовления возможно образование дефектов, загрязнение металла в месте контакта. В этих и многих других случаях бесконтактные методы измерения сопротивления имеют преимущество. Поэтому проблема развития бесконтактных методов измерения удельного электрического сопротивления металлов представляется достаточно актуальной, особенно в области высоких температур.

Бесконтактные методы основаны на использовании электромагнитного поля [1-3]. В работе [1] предложен метод дифференциального трансформатора. Описанный в $[2,3]$ способ состоит в определении угла поворота твердого образца (или ампулы сферической формы с жидким металлом) под воздействием вращающегося магнитного поля. Эти методы в отличие от контактного обременены большим количеством измерительной аппаратуры (особенно, в [2,3]) и довольно сложной процедурой обработки экспериментальных результатов, не говоря уже о сложности создания вращающегося магнитного поля. Поэтому едва ли можно рассматривать их как предпочтительные в сравнении с контактным методом.

В работе [4] нами предложен простой метод бесконтактного измерения удельного электрического сопротивления $\rho(T)$ металла, базирующийся на использовании электромагнитного поля, создаваемого высокочастотным индукционным генератором. Суть метода измерение электродвижущей силы $\varepsilon_{i}$ (ЭДС), наведенной высокочастотным электромагнитным полем в двух круговых контурах диаметрами $d_{c 1}>d_{c 2}>d(d-$ диаметр образца), размещенных коаксиально с образцом в виде 
цилиндра, что позволяет при известной для исследуемого образца связи $\rho=f\left(\varepsilon_{1}, \varepsilon_{2}\right)$ находить значение $\rho$. Для установления этой связи необходимо решить задачу о мощности, введенной в цилиндрический образец при его нагревании в индукторе высокочастотного генератора. В работе [4] эта связь приведена для образца в виде сплошного цилиндра. С целью расширения возможностей метода необходимо найти указанную связь для образца в виде тонкостенного цилиндра, что позволит с помощью предложенного метода измерять $\rho$ листового металла, т.е. предварительно нужно решить задачу о мощности, введенной в тонкостенный цилиндр при его индукционном нагреве.

\section{1. Постановка задачи}

При применении метода измерения $\rho$ в случае листового металла образец должен иметь форму тонкостенного цилиндра с толщиной стенки порядка $0.1-0.5 \mathrm{~mm}$. Для отыскания функции $\rho=f\left(\varepsilon_{1}, \varepsilon_{2}\right)$ используется очевидный с физической точки зрения факт - мощность $W$, введенная в образец, зависит только от напряженности $H$ магнитного поля и электрических свойств материала образца. Поскольку в [4] приведена формула связи в случае сплошного цилиндра, нужно решить задачу о величине мощности, введенной в тонкостенный цилиндр при его нагревании в высокочастотном электромагнитном поле. Тогда, приравнивая выражения для $W$, содержащие величины наведенных на одном витке круговых контуров ЭДС $\varepsilon_{1}$ и $\varepsilon_{2}$, геометрию образца (диаметр d) и контуров (диаметры $d_{c 1}$ и $d_{c 2}$ ), можно найти искомую связь $\rho=f\left(\varepsilon_{1}, \varepsilon_{2}\right)$ в случае тонкостенного цилиндра.

\section{2. Теоретическая часть}

При нагревании металлического образца в высокочастотном электромагнитном поле вводимая в образец мощность выделяется в скин-слое, толщина которого описывается выражением: $\sigma(T)=\left[\rho(T) /\left(\pi \mu_{0} \mu f\right)\right]^{1 / 2}$, где: $\mu_{0}-$ магнитная постоянная, равная $4 \pi \cdot 10^{-7} \mathrm{H} / \mathrm{m}$; $\mu=1-$ магнитная проницаемость материала образца в области высоких температур $\left(T>T_{K}\right), f-$ линейная частота порядка $0.5 \mathrm{MHz}$, характерная для промышленных высокочастотных генераторов.

В случае образца в виде тонкостенного цилиндра толщина его стенки $\Delta R=R_{2}-R_{1}$ будет меньше толщины скин-слоя. На рис. 1 и 2 представлена температурная зависимость $\rho(T)$ и для металлов, сильно отличающихся по величине $\rho(T)$. Из рис. 2 видно, что значения $\sigma(T) \sim[\rho(T)]^{1 / 2}$ лежат в пределах $0.4-0.85 \mathrm{~mm}$. Это означает, что магнитное поле будет проникать в полость цилиндра, толщина стенок которого лежит в пределах $0.1-0.5 \mathrm{~mm}$ даже при самой низкой температуре $\sim 1000 \mathrm{~K}$. Чтобы исключить попадание магнитного поля через торцы цилиндра, они должны быть закрыты

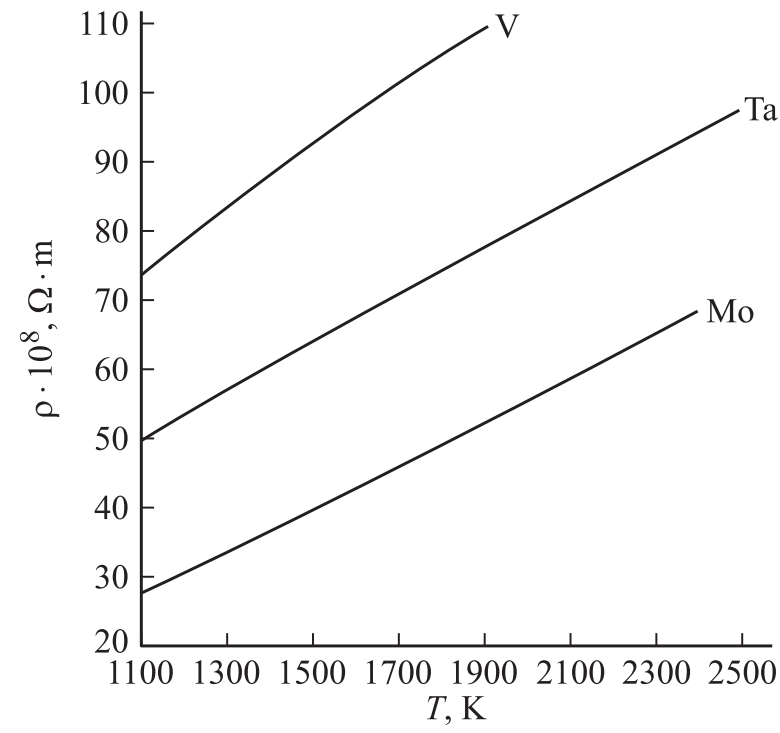

Рис. 1. Зависимость удельного электросопротивления металлов от температуры.

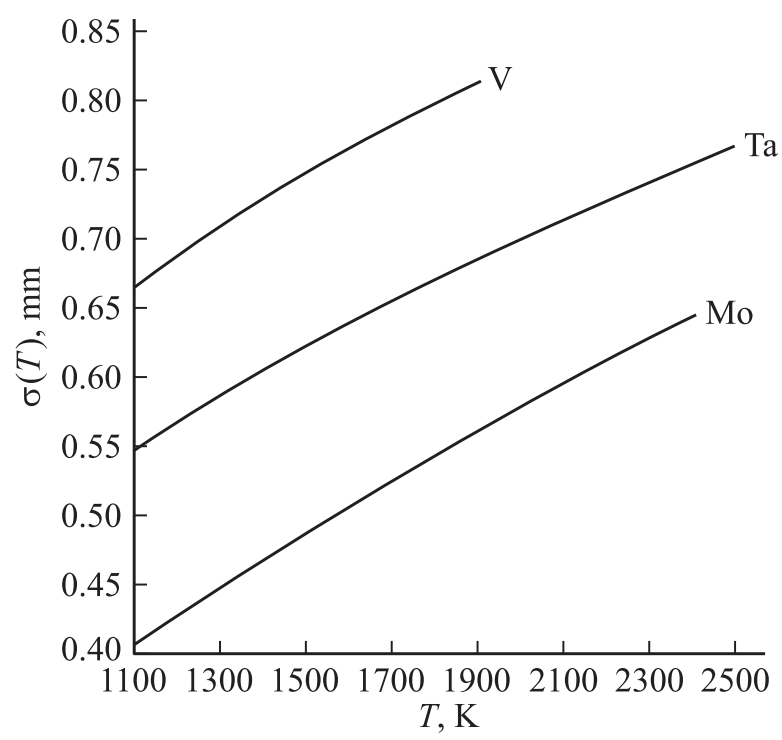

Рис. 2. Зависимость эффективной толщины скин-слоя $\sigma(T)$ от температуры для металлов с разными значениями $\rho(T)$.

металлическими дисками толщиной $\sim 1.5 \mathrm{~mm}$. В результате магнитное поле может проникать внутрь цилиндра только через боковую поверхность.

Вследствие объемного характера тепловыделения при индукционном нагреве необходимо знать функцию распределения внутренних источников энергии:

$$
w(r)=\gamma|E(r) / \sqrt{2}|^{2}, \quad(\gamma=1 / \rho),
$$

тогда поставленная задача сводится к отысканию функции распределения $E(r)$, т.е., решению системы уравнений Максвелла при определенных граничных условиях.

При решении задачи будем считать выполненными следующие предпосылки: 
1) создаваемое индуктором поле обладает осевой симметрией (это условие эквивалентно предположению о больших в сравнении с диаметрами длинах индуктора и образца);

2) зависимость от времени компонент электромагнитного поля является гармонической: $H(r, t)=H(r) e^{i \omega t}$; $E(r, t)=E(r) e^{i \omega t}, \quad($ круговая частота $\omega=2 \pi f)$.

Уравнения Максвелла можно записать в виде дифференциального уравнения:

$$
\frac{d^{2} H(r)}{d r^{2}}+\frac{1}{r} \frac{d H(r)}{d r}+i \omega \mu_{0} \mu \gamma H(r)=0 .
$$

Граничные условия с учетом того, что $\Delta R<\sigma$, будут иметь вид

$$
H\left(R_{2}\right)=H_{0} ;\left.\frac{d H(r)}{d r}\right|_{r=R_{1}}=0 .
$$

Второе граничное условие означает, что $H\left(R_{1}\right)=$ const, т. е. в полости нет затухания, так как образец размещается в вакуумной камере.

Решением уравнения (2) при граничных условиях (3) находятся напряженности магнитного и электрического полей:

$$
\begin{gathered}
H(r, t)=\frac{H_{0} \sqrt{i}}{\Delta}\left[F_{1}(\xi \delta, \xi \delta)+i F_{2}(\xi \delta, \xi \delta)\right] e^{i \omega t}, \\
E(r, t)=\frac{H_{0} \sqrt{2} \rho}{\Delta \sigma} i\left[F_{1}^{\prime}(\xi \delta, \xi \delta)+i F_{2}^{\prime}(\xi \delta, \xi \delta)\right] e^{i \omega t}, \\
\Delta=\sqrt{i}\left[F_{1}(\xi \delta, \delta)+i F_{2}(\xi \delta, \delta)\right] .
\end{gathered}
$$

При записи (4) введены следующие обозначения: $\xi=r / R_{2} \quad(r-$ текущая координата $) ; \quad \xi=R_{1} / R_{2}$; $\delta=R_{2} \sqrt{2} / \sigma ; F_{j}(j=1,2)$ - действительная и мнимая части разности произведений цилиндрических функций Бесселя и Ганкеля нулевого и первого порядков (и их производных $\left.F_{j}^{\prime}=d F_{j} / d \xi\right)$ ):

$$
\begin{aligned}
& \sqrt{i}\left[F_{1}(\xi \delta, \xi \delta)+i F_{2}(\xi \delta, \xi \delta)\right]= \\
& =\left[H_{1}^{(1)}(\xi \delta \sqrt{i}) J_{0}(\xi \delta \sqrt{i})-J_{1}(\xi \delta \sqrt{i}) H_{0}^{(1)}(\xi \delta \sqrt{i})\right] .
\end{aligned}
$$

Для нахождения функции $w(r)$ в случае полого цилиндра достаточно в (1) подставить эффективное значение напряженности электрического поля в стенке цилиндра. Используя вторую формулу в (4), найдем

$$
w(r)=\frac{\rho H_{0}^{2}}{\sigma^{2}} \frac{\left[F_{1}^{\prime}(\xi \delta, \xi \delta)\right]^{2}+\left[F_{2}^{\prime}(\xi \delta, \xi \delta)\right]^{2}}{|\Delta|^{2}} .
$$

Выделившуюся в образце мощность получим подстановкой (6) в формулу (1) и последующим ее интегрированием в пределах от $R_{1}$ до $R_{2}$ :

$$
\begin{aligned}
W & =2 \pi \int_{R_{1}}^{R_{2}} w(r) r d r=\pi \delta \rho H_{0}^{2}\left[F_{1}(\xi \delta, \delta) F_{1}^{\prime}(\xi \delta, \delta)\right. \\
& \left.\left.+F_{2}(\xi \delta, \delta) F_{2}^{\prime}(\xi \delta, \delta)\right] / F_{1}^{2}(\xi \delta, \delta)+F_{2}^{\prime}(\xi \delta, \delta)\right] .
\end{aligned}
$$

Формула (7) позволяет вычислить мощность нагрева, если известна величина напряженности магнитного поля $H_{0}$. Для ее определения используем связь между $H_{0}$ и легко измеряемой величиной электродвижущей силы $\varepsilon$, наводимой в круговом контуре радиусом $R_{c}$, охватывающем образец, как это сделано в [5] для случая сплошного цилиндра. При сделанных выше предположениях о характере поля, ЭДС индукции, наведенная в контуре радиусом $R_{c}$, будет равна

$$
\begin{aligned}
\varepsilon_{i}=-d \Phi / d t= & \pi i \omega \mu_{0}\left[H_{0}\left(R_{c}^{2}-R_{2}^{2}\right)\right. \\
& \left.+2 \int_{R_{1}}^{R_{2}} H(r) r d r+R_{1}^{2} H\left(R_{1}\right)\right], \quad[\mathrm{V}],
\end{aligned}
$$

где $\Phi(r)=B(r) S-$ поток магнитной индукции $B(r)=\mu_{0} H(r)$ через площади $S_{i}(i=1,2,3)$ соответствующих поверхностей; $H\left(R_{1}\right)$ - модуль входящего в (4) выражения для напряженности магнитного поля, в котором положено $r=R_{1}: H\left(R_{1}\right)=2 H_{0} / \pi \delta_{1} \Delta_{1}$.

Дальнейшие выкладки, которые здесь опущены, заключаются в следующем. Величина $H_{0}^{2}$, выраженная после интегрирования (8) - через модуль $\left|\varepsilon_{i}\right|^{2}$, подставляется в (7). Полученное соотношение упрощается путем замены цилиндрических функций от мнимого аргумента функциями Томсона от действительного аргумента с последующим их разложением в ряд по параметру $\beta=\Delta R / R_{2}=(1-\zeta) \ll 1$. В итоге получается формула для мощности, введенной в тонкостенный цилиндр с торцами $\left(N_{c i}=d_{c i} / d ; \eta=\sigma / d-\right.$ безразмерная толщина скин-слоя):

$$
\begin{aligned}
W= & \left(2 \varepsilon_{i}^{2} / \pi \rho \beta^{3}(1+\beta / 2) /\left\{\left[\left(1+\beta+\beta^{2}\right) N_{c i}^{2}\right]^{2}\right.\right. \\
& \left.+\left[\left(N_{c i}^{2}-1\right)(\beta / 2 \eta)^{2}\right]^{2}\right\},[\mathrm{W} / \mathrm{cm}] .
\end{aligned}
$$

Приравнивая выражения (9), записанные для разных значений $N_{c i}(i=1,2)$, получим искомую формулу, связывающую отношение $\varepsilon_{1} / \varepsilon_{2}$ и величину $\eta$ :

$$
\begin{gathered}
\left(\varepsilon_{1} / \varepsilon_{2}\right)^{2}=\left\{\left[\left(1+\beta+\beta^{2}\right) N_{c 1}^{2}\right]^{2}+\left[\left(N_{c 1}^{2}-1\right)(\beta / 2 \eta)^{2}\right]^{2}\right\} \\
/\left\{\left[\left(1+\beta+\beta^{2}\right) N_{c 2}^{2}\right]^{2}+\left[\left(N_{c 2}^{2}-1\right)(\beta / 2 \eta)^{2}\right]^{2}\right\} .
\end{gathered}
$$

В формуле (10) неизвестными являются $\eta$ и отношение ЭДС $\varepsilon_{1} / \varepsilon_{2}$. Для работы с экспериментальными данными предварительно рассчитывается правая часть формулы (10), содержащая значения $\eta$ из диапазона $0.03-0.1$ с шагом 0.005 при заданных: геометрии образца $-d$, $\Delta R$, и величин $d_{c i}$. Затем строится график зависимости $\eta=f\left(\varepsilon_{1} / \varepsilon_{2}\right)$. Заметим, что с физической точки зрения отношение ЭДС зависит от $\eta$, т.е. $\varepsilon_{1} / \varepsilon_{2}=f(\eta)$. Однако в графическом виде удобнее строить зависимость $\eta=f\left(\varepsilon_{1} / \varepsilon_{2}\right)$, так как в эксперименте определяется отношение ЭДС, по которому и находится величина $\eta$ и тем самым $\rho(T)=4 \pi^{2} d^{2} f(T)[\eta(T)]^{2}$. 
В случае тонкостенного цилиндра, например, при $d$. $=12 \mathrm{~mm}, d_{c 1}=22 \mathrm{~mm}$ и $d_{c 2}=19 \mathrm{~mm}$ функциональная зависимость $\eta=f\left(\varepsilon_{1} / \varepsilon_{2}\right)$ описывается полиномом 4-й степени и носит ярко выраженный нелинейный характер $\left(x=\varepsilon_{1} / \varepsilon_{2}\right)$ :

$$
\begin{aligned}
\eta(x)=0.3525 x^{4}- & 2.3247 x^{3}+5.6275 x^{2} \\
- & 6.0395 x+2.5282 ; R^{2}=1 .
\end{aligned}
$$

В случае сплошного цилиндра аналогичная зависимость описывается практически линейной функцией.

\section{3. Экспериментальная часть}

Экспериментальная отработка метода осуществлялась на установке, схема которой представлена на рис. 3 . Образец 1 в виде тонкостенного цилиндра с торцами вместе с катушкой 3 , расположенной на 5-6 mm ниже модели черного тела 2, вставляется в находящийся в вакуумной камере индуктор (не показан, как и элементы креплений) высокочастотного генератора, и создается вакуум. Абсолютная и яркостная температуры определяются по модели черного тела 2 и светимости поверхности образца пирометром (не показан), и с помощью переключателя П поочередно вольтметром $V$ замеряются ЭДС, наведенные на секциях и на их сумме.

Для повышения точности измерений проволочный контур, наматываемый на керамическую катушку, состоял из двух секций, содержащих 4 и 3 витка, снабженных выводами. Это позволило измерять наведенную ЭДС на каждой секции и на их сумме, и находить среднее значения $\bar{\varepsilon}=\left(\varepsilon_{1}+\varepsilon_{2}+\varepsilon_{3}\right) / 3$. Так как высота двухсекционной керамической катушки как минимум в два раза больше высоты односекционной катушки, поэтому во избежание влияния на температурное поле образца керамического изделия из двух двухсекционных катушек эксперимент повторялся дважды: с одной катушкой диаметром $d_{c 1}$, а затем с другой диаметром $d_{c 2}<d_{c 1}$.

Диаметры контуров задавались исходя из механических свойств керамики БГП-10, так, чтобы толщина стенки катушки $\Delta R_{c}=1.5 \mathrm{~mm}$. Только при такой толщине стенки обеспечивается механическая прочность катушки при высоких температурах.

Эксперимент осуществлялся на установке, содержащей высокочастотный генератор ВГТ7-15/440 (мощность $15 \mathrm{~kW}$, частота $440 \mathrm{kHz}$; на самом деле частота генератора зависит от задаваемой мощности генератора, уменьшаясь при ее увеличении). Индуктор генератора введен в вакуумную камеру.

Образец представлял собой тонкостенный цилиндр длиной $100 \mathrm{~mm}$, внешним диаметром $12 \mathrm{~mm}$ и внутренним $11 \mathrm{~mm}(\beta=0.083)$, материал образца - ниобий. Торцы цилиндра закрывались дисками из ниобия толщиной $1.5 \mathrm{~mm}$ для того, чтобы магнитное поле проникало в полость образца только через боковую поверхность цилиндра. При открытых торцах метод не может быть

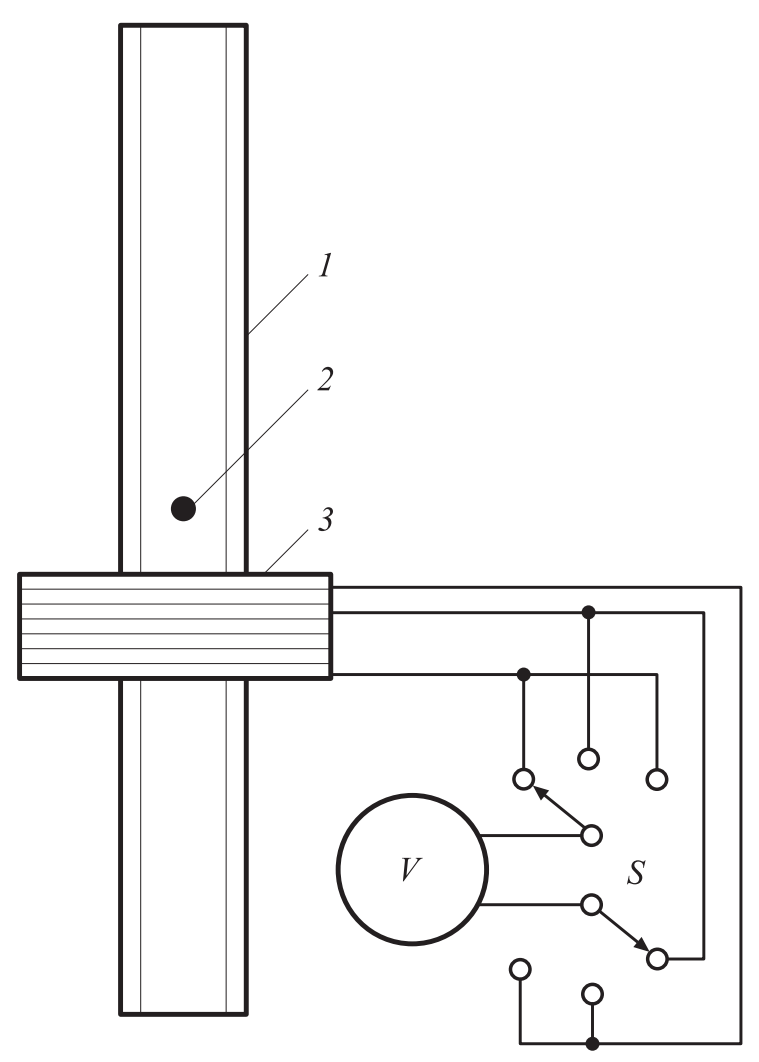

Рис. 3. Схема установки (индуктор и детали крепления не показаны): 1 - металлический тонкостенный образец цилиндрической формы (диаметр 10-12 mm, длина $80-100 \mathrm{~mm}$, толщина стенки - $(0.1-0.5) \mathrm{mm}) ; 2-$ модель черного тела; 3 - двухсекционная катушка диаметром $d_{c i}$ с разным числом витков в секциях; $S$ - трехсекционный сдвоенный переключатель; $V$ - вольтметр.

реализован. Образец вместе с катушкой помещался в индуктор, и в камере создавался вакуум на уровне $10^{-3} \mathrm{~Pa}$.

Эксперимент проводился следующим образом. По достижении вакуума образец прокаливался при температуре $\sim 1700 \mathrm{~K}$ в течение $\sim 2 \mathrm{~h}$. Затем устанавливалась минимально возможная температура, и пирометром измерялись абсолютная и яркостная температуры, а с помощью вольтметра замерялись ЭДС индукции, наведенные на секциях катушки и на их сумме. Частота генератора показывалась на его дисплее. Затем изменением мощности генератора устанавливалась новая температура, и процедура повторялась вплоть до достижения максимальной температуры. По ее достижении эксперимент проводился в обратном порядке. По описанной схеме эксперимент повторялся с другой двухсекционной катушкой.

\section{4. Методика обработки результатов эксперимента}

Так как температуры в обоих экспериментах не совпадали, это усложнило обработку его результатов, по- 
Таблица 1. Экспериментальные усредненные данные

\begin{tabular}{c|c|c|c|c|c|c|c}
\hline$T, \mathrm{~K}$ & $f, \mathrm{kHz}$ & $\bar{\varepsilon}_{1}(T), \mathrm{V}$ & $\bar{\varepsilon}_{2}(T), \mathrm{V}$ & $\bar{\varepsilon}_{1} / \bar{\varepsilon}_{2}$ & $\eta\left(\bar{\varepsilon}_{1} / \bar{\varepsilon}_{2}\right)$ & $\rho \cdot 10^{8} \Omega \cdot \mathrm{m}$ & $\rho_{\mathrm{Nb}} \cdot 10^{8} \Omega \cdot \mathrm{m}$ \\
\hline 1100 & 438.4 & 4.091 & 2.883 & 1.36645 & 0.06386 & 50.8 & 49.0 \\
1200 & 438.0 & 5.142 & 3.792 & 1.35542 & 0.06580 & 53.9 & 51.9 \\
1300 & 437.0 & 6.354 & 4.719 & 1.34562 & 0.06773 & 56.9 & 54.9 \\
1400 & 435.4 & 7.743 & 5.769 & 1.33691 & 0.06967 & 60.0 & 57.6 \\
1500 & 432.2 & 9.311 & 7.004 & 1.32921 & 0.07162 & 63.1 & 60.9 \\
1600 & 430.4 & 11.092 & 8.386 & 1.32244 & 0.07358 & 66.2 & 63.8 \\
1700 & 427.0 & 13.070 & 9.928 & 1.31652 & 0.07557 & 69.2 & 66.8
\end{tabular}

Таблица 2. Результаты расчета мощности $W\left(\bar{\varepsilon}_{i}\right) / \mathrm{cm}$ и степеней черноты $\varepsilon_{T}(T)$ и $\varepsilon_{\lambda T}(T)$

\begin{tabular}{c|c|c|c|c|c|c|c|c|c}
\hline$T, \mathrm{~K}$ & $T_{S}, \mathrm{~K}$ & $W\left(\bar{\varepsilon}_{1}\right) / \mathrm{cm}$ & $W\left(\bar{\varepsilon}_{2}\right) / \mathrm{cm}$ & $\varepsilon_{T}\left(\bar{\varepsilon}_{1} T\right)$ & $\varepsilon_{T}\left(\bar{\varepsilon}_{2} T\right)$ & $\varepsilon_{T}(T)_{\mathrm{Nb}}$ & $\varepsilon_{\lambda T}(T)$ & $\varepsilon_{\lambda T}(T)_{\mathrm{Nb}}$ & $\sigma(T), \mathrm{mm}$ \\
\hline 1100 & 1055 & 3.53 & 3.46 & 0.123 & 0.121 & 0.118 & 0.425 & - & 0.756 \\
1200 & 1145 & 5.89 & 5.83 & 0.133 & 0.132 & 0.128 & 0.409 & - & 0.790 \\
1300 & 1233 & 8.67 & 8.58 & 0.142 & 0.141 & 0.137 & 0.397 & 0.384 & 0.813 \\
1400 & 1321 & 12.42 & 12.30 & 0.151 & 0.150 & 0.147 & 0.386 & 0.381 & 0.836 \\
1500 & 1407 & 17.35 & 17.18 & 0.160 & 0.159 & 0.156 & 0.377 & 0.378 & 0.859 \\
1600 & 1492 & 23.79 & 23.56 & 0.170 & 0.168 & 0.165 & 0.369 & 0.375 & 0.883 \\
1700 & 1577 & 31.94 & 31.63 & 0.179 & 0.177 & 0.174 & 0.362 & 0.372 & 0.907 \\
\hline
\end{tabular}

Отклонение от данных для $\mathrm{Nb}$ в \%

$\left[\varepsilon_{T}\left(\bar{\varepsilon}_{1} T\right) / \varepsilon_{T}(T)_{\mathrm{Nb}}\right] \%=4.2-2.8 ; \quad\left[\varepsilon_{T}\left(\bar{\varepsilon}_{2} T\right) / \varepsilon_{T}(T)_{\mathrm{Nb}}\right] \%=2.8-1.8$.

$\left[\varepsilon_{\lambda T}(T) / \varepsilon_{\lambda T}(T)_{\mathrm{Nb}}\right] \%=2.7-3.4$.

Пр и ме чан и е. В нижней строке таблицы показано отклонение в \% экспериментальных значений степеней черноты от степеней черноты ниобия $(\mathrm{Nb})$, взятых из работы [5] и приведенных в соответствующих столбцах таблицы, т. е., это $\left[\varepsilon_{T}\left(\bar{\varepsilon}_{1} T\right) / \varepsilon_{T}(T)_{\mathrm{Nb}}\right] \%$ и $\left[\varepsilon_{T}\left(\bar{\varepsilon}_{2} T\right) / \varepsilon_{T}(T)_{\mathrm{Nb}}\right] \%$. То же самое для спектральной степени черноты (рядом стоящие столбцы): $\left[\varepsilon_{\lambda T}(T) / \varepsilon_{\lambda T}(T)_{\mathrm{Nb}}\right] \%$.

скольку все измеренные величины необходимо отнести к одной температуре. Для этого строились графики: $f(T)$; предварительно усредненных значений $\bar{\varepsilon}_{1}(T)$ и $\bar{\varepsilon}_{2}(T)$; находились их трендовые функции с величиной достоверности аппроксимации $R^{2} \sim 1$. Затем задавалась температурная шкала $1100-1700 \mathrm{~K}$ с шагом 100, включающая все экспериментальные температуры, и по найденным трендовым функциям находились значения $f(T), \bar{\varepsilon}_{1}(T)$ и $\bar{\varepsilon}_{2}(T)$. Затем определялось отношение $\bar{\varepsilon}_{1}(T) / \bar{\varepsilon}_{2}(T)$, которое вставлялось в заранее построенную аппроксимирующую функцию $\eta=f\left(\bar{\varepsilon}_{1} / \bar{\varepsilon}_{2}\right)$, что позволяло найти значения удельного электрического сопротивления - $\rho(T)=4 \pi^{2} d^{2} f(T)[\eta(T)]^{2}$ Результаты экспериментов представлены в табл. 1 .

Отличие полученных значений удельного электрического сопротивления ниобия от данных [5] не превосходит $\sim 3 \%$. Это можно объяснить как отличием материала тонкостенного образца от содержащего меньше примесей чистого ниобия, так и разными величинами погрешностей контактного и предлагаемого методов.

Следует отметить, что формула (9) позволяет рассчитать вводимую в образец мощность, поскольку все входящие в нее переменные теперь определены. Результаты расчета мощностей $W(\bar{\varepsilon}, T)$ представлены в табл. 2. Здесь же приведены значения яркостной температуры, необходимые для расчета величины полусферической монохроматической $(\lambda=0.65 \mu \mathrm{m})$ спектральной степени черноты $\varepsilon_{\lambda T}(T)$.
Для подтверждения объективности полученных данных свойства материала - ниобия - сопоставлялись с данными [5]. Для этого рассчитывались полусферические интегральные степени черноты по значениям полученных с помощью формулы (9) мощностей, и найденная по значениям температур - абсолютной и яркостной полусферическая монохроматическая спектральная степень черноты. Результаты расчетов сведены в табл. 2, в последней строке которой указаны отклонения экспериментальных данных от приведенных в [5]. В последнем столбце таблицы приведены значения толщины скинслоя $\sigma(T)$ в миллиметрах. Видно, что она действительно больше толщины стенки цилиндра, равной $0.5 \mathrm{~mm}$.

Сопоставление по столбцам таблицы показывает, что различия между значениями полусферической интегральной степени черноты, полученные при разных диаметрах $-d_{c 1}$ и $d_{c 2}-$ круговых контуров, и данными для ниобия, в первом случае лежат в диапазоне $(4.2-2.8) \%$, а во 2-м - в диапазоне $(2.8-1.8) \%$. Следует отметить, что значения полусферической интегральной степени черноты зависят от класса обработки поверхности исследуемого образца. Поскольку в обоих экспериментах использовался один и тот же образец, то различия в экспериментальных значениях интегральной степени черноты следует объяснить завышением значений $\bar{\varepsilon}_{1}(T)$, и тем самым вводимой в образец мощности $W(\bar{\varepsilon}, T)$. Отмеченные бо́льшие отличия в эксперименте с $d_{c 1}$ свидетельствуют о наличии влияния радиальной неравномерности 
магнитного поля при диаметре контура $d_{c 1}=22 \mathrm{~mm}$. Поэтому его не следует увеличивать в целях получения бо́льших значений $\bar{\varepsilon}_{1}(T)$. Этот вывод относится к геометрии: индуктора, диаметра кругового контура и образца: $D_{\text {ind. }}=40 \mathrm{~mm}, d_{c 1}=22 \mathrm{~mm}, d=12 \mathrm{~mm}$. При другом диаметре $D_{\text {ind. }}$ геометрия остальных элементов будет другая. Установить, какая именно, следует экспериментально. Отметим, что предложенный метод позволяет осуществить внутренний контроль объективности экспериментальных данных путем сопоставления результатов расчета мощности по величинам ЭДС, наведенных на круговых контурах разных диаметров, которые в пределах погрешности должны совпадать.

\section{Заключение}

Приведенный материал позволяет сформулировать следующее заключение. Результаты экспериментов, полученные для ниобия в виде тонкостенного цилиндра с использованием бесконтактного метода, мало отличаются от данных для сплошного цилиндра, полученных при использовании стандартного контактного метода. Учитывая, что экспериментальное осуществление бесконтактного метода отличается исключительной простотой, он может быть рекомендован для исследования электрических свойств листового металла в качестве предпочтительного, особенно в области высоких температур.

\section{Конфликт интересов}

Авторы заявляют, что у них нет конфликта интересов.

\section{Список литературы}

[1] Бутыгин Ю.В., Гнатов А.В., Барбашова М.В., Гаврилова T.B., Степанов A.A. // Електротехніка і Електромеханіка. 2012. № 1. C. 69.

[2] Поводатор А.М., Вьюхин В.В., Цепелев В.С. Способ и устройство для бесконтактного измерения удельного электрического сопротивления металлического сплава методом вращающегося магнитного поля. Патент № 2531056 РФ. Бюл. № 29. Опубл. 20.10.2014.

[3] Кононенко В.И., Ражкабов А.А., Рябина А.В. // Расплавы. 2009. № 1. C. 36.

[4] Румянцев А.B. Способ и устройство для бесконтактного определения удельного электросопротивления металлов в области высоких температур. Патент № 2687504 РФ. Бюл. № 14. Опубл. 14.05.2019.

[5] Макаренко И.Н. Исследование теплопроводности и теплоемкости твердых металлов в области высоких температур 1100-2500 К. Дисс. канд. физ.-мат.наук. Физический факультет МГУ им. М.В. Ломоносова. 1970. 